\title{
Crack branching instability and directional stability in dynamic fracture of a tough bulk metallic glass
}

\author{
M.Q. Jiang ${ }^{\text {a,b }}$, J.X. Meng ${ }^{\text {c,d }}$, V. Keryvin ${ }^{\mathrm{d}}$, L.H. Dai ${ }^{\mathrm{a}, \mathrm{b}, *}$ \\ a State Key Laboratory of Nonlinear Mechanics, Institute of Mechanics, Chinese Academy of Sciences, Beijing 100190, People's Republic of China \\ ${ }^{\mathrm{b}}$ State Key Laboratory of Explosion Science and Technology, Beijing Institute of Technology, Beijing 100081, People's Republic of China \\ ${ }^{\mathrm{c}}$ Applied Mechanics Laboratory of the University of Rennes 1, LARMAUR, ERL CNRS 6274, Campus de Beaulieu, 35042 Rennes cedex, France \\ ${ }^{\mathrm{d}}$ Department of Materials Engineering, LIMATB, EA4250, Université de Bretagne-Sud, Centre de recherche de St Maudé, 56321 Lorient cedex, France
}

\section{A R T I C L E I N F O}

\section{Article history:}

Received 23 April 2011

Received in revised form

15 July 2011

Accepted 26 July 2011

Available online 28 August 2011

\section{Keywords:}

B. Brittleness and ductility

B. Fracture mode

B. Glasses, metallic

\begin{abstract}
A B S T R A C T
We report the ductile fracture behavior of a tough Zr-based bulk metallic glass (BMG) in the dynamic regime, where a rapid moving crack initiates by a Mode II shearing and then advances through plastic voids growth and linking. A clear fractographic evolution from river-like vein pattern to chevron-like zone where microscopic dimples and voids coexist, followed by crack microbranching pattern, was observed on the dynamic fracture surfaces. A terminal crack velocity of about 0.5 of the Rayleigh wave velocity was determined for the onset of such microbranching instability. Furthermore, the dynamic branched daughter cracks are favored to continue in their original mother crack plane, having "directional stability". Our findings may increase the understanding of the dynamic fracture of BMGs with both glassy nature and metallic bonding character.
\end{abstract}

(c) 2011 Elsevier Ltd. All rights reserved.

\section{Introduction}

The dynamic behavior of crack propagation in brittle materials continues to fascinate scientists because of both fundamental and practical importance [1-6]. In such studies, a very basic question is how the system chooses to dispose of the externally imposed energy. In conventional brittle materials, in terms of poor toughness (e.g., soda-lime glass and PMMA), the propagating crack converts the fracture energy flowing into its tip to the new surface(s); the resulting fracture pattern is described as 'mirror, mist and hackle': an initially featureless mirror-like surface begins to appear misty and then evolves into a rough hackle region [1-4]. Bulk metallic glasses (BMGs) usually exhibit a brittle behavior (like a glass) at the macroscopic level but a ductile behavior (as a metal) at the microscopic level [7-12], except for some specially-designed compositions [13-15]. Particularly, self-organized nanoscale periodic corrugations (NPCs) have been widely observed on the socalled 'mirror' regions in brittle BMGs [5,11,16-18] or even some tough systems subjected to very high-strain-rate loading [19-24]. It has been proposed that the NPC forms via tension-

\footnotetext{
* Corresponding author. State Key Laboratory of Nonlinear Mechanics, Institute of Mechanics, Chinese Academy of Sciences, Beijing 100190, People's Republic of China. Tel.: +86 10 82543958; fax: +8610 82543977 .

E-mail addresses: mqjiang@imech.ac.cn (M.Q. Jiang), lhdai@lnm.imech.ac.cn (L.H. Dai).
}

transformation-zone (TTZ)-mediated quasi-cleavage with meniscus instability mode of local plastic flow in the background ahead of the crack tip [19,21-24]. It is also noted that cavitation or void growth and coalescence is generally suggested to explain the dynamic fracture of BMGs $[20,25,26]$. In this paper, we develop a dynamic forced-shear method of hat-shaped specimens, and succeed in achieving Mode I cracking of a typical tough $\mathrm{Zr}$-based BMG at imposed strain rates of about $10^{3} \mathrm{~s}^{-1}$. It is found that the dynamic fracture occurs cooperatively by both the meniscus instability and cavitation mechanisms, followed by crack branching instability. The underlying fracture mechanism is briefly discussed as well.

\section{Experimental}

We choose a typical tough $\mathrm{Zr}_{41.2} \mathrm{Ti}_{13.8} \mathrm{Cu}_{10} \mathrm{Ni}_{12.5} \mathrm{Be}_{22.5}$ (Vit 1) $\mathrm{BMG}$ as a model material, whose fracture toughness $K_{\mathrm{IC}}$ is greater than $50 \mathrm{MPa} \sqrt{\mathrm{m}}[21,27-30]$. Master alloy ingots were obtained by arc-melting the elements $\mathrm{Zr}, \mathrm{Ti}, \mathrm{Cu}, \mathrm{Ni}$ and Be with a purity of $99.9 \%$ or better together under a Ti-gettered $\mathrm{Ar}$ atmosphere. To ensure homogeneity, the master alloy ingots were re-melted several times and subsequently suction-drawn into copper molds to form rods $8 \mathrm{~mm}$ in diameter and $\sim 80 \mathrm{~mm}$ in length. The glassy structure of rods was confirmed by X-ray diffraction in a Philips PW 1050 diffractometer using $\mathrm{CuK} \alpha$ radiation. Hat-shaped specimens (see 

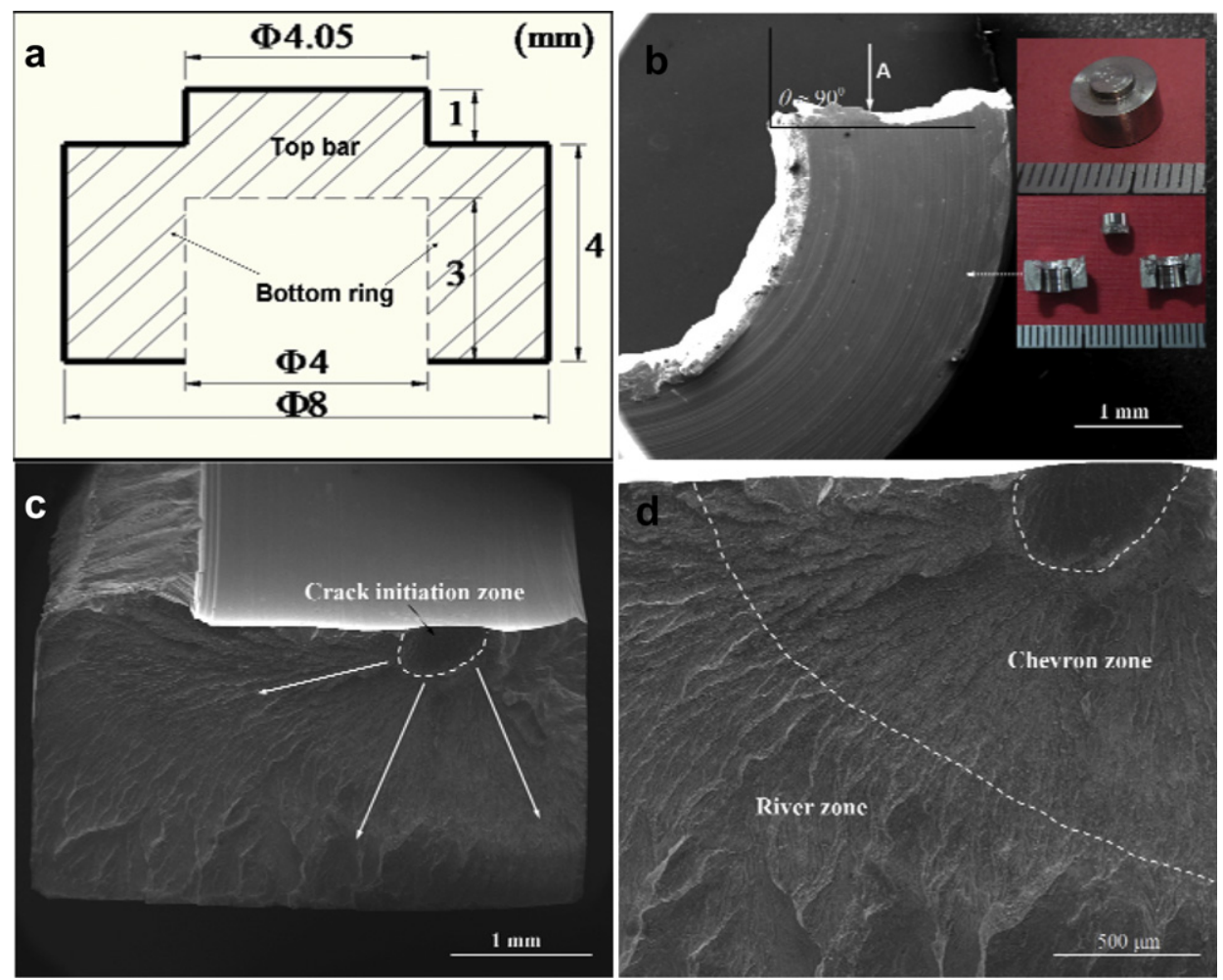

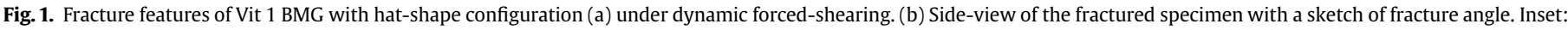
real specimen before and after testing. (c and d) Fracture surface at different magnification marked by an arrow " $A$ " in (b).

Fig. 1a) with specially-designed dimensions were obtained by lathe machining the as-cast Vit 1 rods with using coolant. Dynamic forced-shear experiments were performed with a split Hopkinson pressure bar (SHPB) at room temperature. The hat-shaped specimen was sandwiched between the incident and the transmitted bars. The launched bullet at the speed of $\sim 20 \mathrm{~m} / \mathrm{s}$ was impacted onto the free end of the incident bar. At the moment of impact, the generated elastic-wave in the incident bar compresses the top bar of specimen that is displaced into the bottom ring, which induces a circumferential tensile stress in the bottom ring. Once the circumferential tensile stress exceeds the tensile strength of Vit 1, it thus leads to a mode-I crack propagating along the radial direction which finally separates the bottom ring of the sample into two or more parts (see the insert to Fig. 1b). The Mode I fracture surfaces were characterized by a FEI Sirion high-resolution scanning electron microscopy (HRSEM) with spatial resolution of $1.5 \mathrm{~nm}$.

\section{Results and discussion}

Fig. 1b shows the macroscopic side-view of the Mode I fracture surface. It was found that the intersection angle between the fracture plane and the hoop stress is approximately $90^{\circ}$, which indicates that the circumferential tensile stress plays a crucial role in the cracking propagation. In addition, the relatively flat fracture surface implies that whole cracking process is approximately in plane-strain state [31]. The top-view of the fracture surface, marked by arrow " $A$ " in Fig. 1b, is presented in Fig. 1c and d at different magnifications. It can be seen that there is a clear crack initiation zone that is relatively smooth, as marked in Fig. 1c. This indicates that the present Mode I crack nucleates at the internal free-surface of the specimen, rather than within its interior. Fig. 1d displays a fractographic transition along the crack propagation direction (as the arrow illustrated in Fig. 1c). The smooth crack initiation zone evolves into a rough chevron zone, finally followed by an even rougher river zone. At the macroscopic scale, the present fracture surface morphology (see Fig. 1c) is very similar to the classical "mirror-mist-hackle" sequence. However, at the microscopic scale, the fracture mechanism is quite distinct, which we will see shortly.

The characteristic fractographies of the three representative zones, i.e., crack initiation zone, chevron zone, and river zone, are clearly shown in Fig. 2a-f, respectively. Very interestingly, the crack initiation zone displays typical vein-like pattern (see Fig. 2a). A close-up view of the area "I" in Fig. 2a is presented in Fig. 2b. It can be seen from this picture that the characteristic length scale of vein pattern, i.e., the ridge spacing, is about $5 \mu \mathrm{m}$. Such river-like vein patterns are so ubiquitous in shear-fracture surfaces of metallic glasses in plane-strain tension [11,32,33]. The observations demonstrate that the current dynamic fracture initiates by a Mode II shearing, although a tensile stress intervenes. Similar phenomenon was also observed by Raghavan et al. [21] in the impact toughness tests on notched annealed Vit 1 BMGs and by Tandaiya et al. [34] in quasi-static four-point bending on notched as-cast Vit 1 BMGs. Based on our previously proposed ductile-to-brittle transition (DBT) criterion [19], the characteristic scales $(\sim 5 \mu \mathrm{m})$ of vein patterns are greater than the critical wavelength $(\sim 85 \mathrm{~nm})$ of the fluid meniscus instability of the present Vit 1 BMGs. Therefore, the meniscus instability plays a dominant role in the initiation process of the dynamic Mode I crack. In this stage, the fracture energy dissipates mainly by viscous/plastic flow in front of the crack tip $[19,35,36]$. At the outside boundary of crack initiation zone, the fracture pattern exhibits a sharp transition from vein-like pattern (see Fig. 2a) to chevron-like pattern (see Fig. 2c). Fig. 2d is a highmagnification micrograph of an area, marked "II" in Fig. 2c. Note that, in addition to chevron-like ridges, microscopic dimples and even micro-voids (circled in Fig. 2d) can be clearly observed. These features are characteristic of ductile fracture. The former implies 

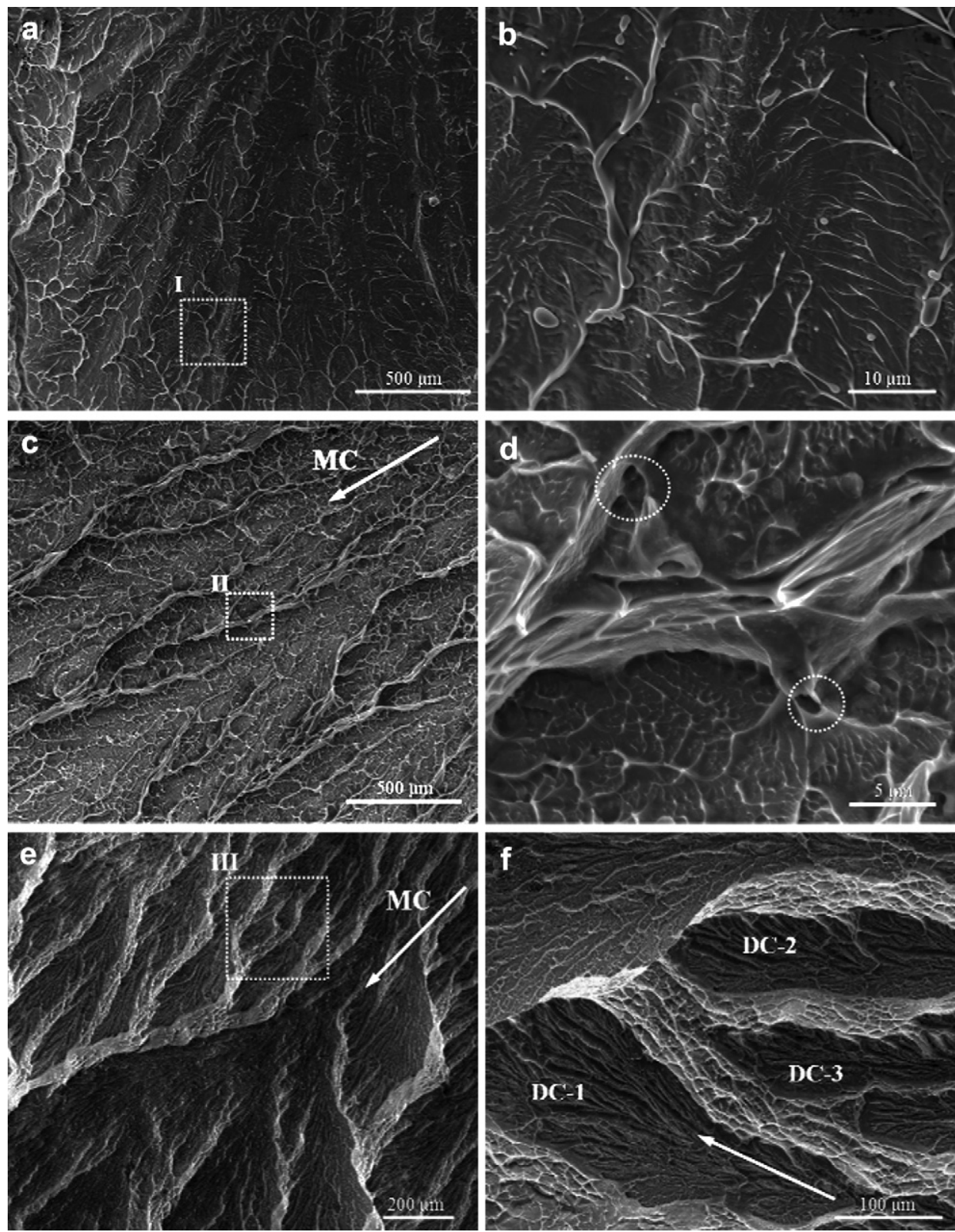

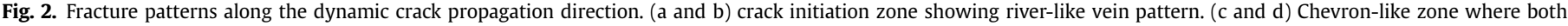
dimples and voids dominate. (d and f) River zone showing crack-microbranching.

that the fluid meniscus instability still occurs in this cracking stage. The latter reveals that cavitation ahead of the crack tip is activated, which should result from the enhanced negative pressure induced by the increase in the crack propagation speed. The similar chevron-like pattern zone has been recently observed on the spall planes of a Zr-based BMG under plate impact experiments [24], where however dimples at the $100 \mathrm{~nm}$ scale appear between deep ridges. With the further increase of the crack propagation speed, the mother crack (MC) finally sprouts many daughter cracks (DCs). Consequently, a river zone appears as shown in Fig. 2e. In this zone, crack-branching together with chevron-like patterns becomes dominant morphology (see Fig. 2f, i.e., the magnified area of "III" in Fig. 2e). It is important to point out that the microbranching promotes the increased fracture energy dissipation of the fast moving dynamic crack and produces more fracture surfaces. The observations provide powerful evidence that the crack branching instability can still afflict the ductile fracture of BMGs in the dynamical regime, which usually occurs in the dynamic brittle fracture of some materials [1-4].

The crack initiation zone marked in Fig. 1c can be considered as a single edge pre-crack that forms due to the fluid meniscus instability. The pre-crack then undergoes dynamic fracture accompanied with a continuous void growth process in a semiinfinite body. In the framework of the linear elastic fracture mechanics (LEFM) [31,37] and further assuming that the voids form due to the coalescence of free volume within the sample [38], we have previously derived the evolution of the crack velocity $v$ along the crack propagation direction, given by [20]

$\frac{v}{v_{R}}=1-\left(\frac{s(v) \mu E}{2.51 \Omega \sigma^{2} \pi l}\right)^{1 / 3}$,

where $v_{R}$ is Rayleigh wave velocity, $s(v)$ is taken as the half of characteristic size of the fracture patterns, $\mu$ is the free volume 
energy chemical potential about $10^{-18} \mathrm{~J}$ [38], $E$ is Young's modulus of 96 GPa [29], $\Omega$ is the average atomic volume $\sim 16.4 \AA^{3}$ [39], $\sigma$ is the remote tensile stress, and $l$ is the crack propagation distance from the sample edge. For the present dynamic fracture of Vit 1 BMG, $s(v) \sim 1 \mu \mathrm{m}$ and $\sigma \approx 2 \mathrm{GPa}$ based on the observation of typical fracture patterns (see Figs. 2c, d and Fig. 2 in Ref. [39]). It can be seen from Fig. 1d that the initial crack length is about $500 \mu \mathrm{m}$. This value is larger than that obtained by Ramamurty group [21,34] who used notched specimens. The main reason is that the notch could significantly facilitate the initiation of a crack. In the present work however, an unnotched specimen was adopted.

After calculating, we get the evolution of $v / v_{R}$ of the dynamic crack after its initiation, which is shown in Fig. 3. We find that at the stage of chevron zone, $v / v_{R}$ increases from 0.28 to about 0.50 . The lower boundary $v / v_{R}=0.28$ is actually the terminal velocity of mode II cracking at the stage of fracture origin (marked in Fig. 1c). The crack branching site $(l \approx 1500 \mu \mathrm{m})$ is just corresponding to a terminal velocity, $v_{b}$, of about $0.5 v_{R}$. The critical velocity $v_{b} /$ $v_{R} \approx 0.5$ is very close to our previously predicted value of $\sim 0.52$ [20]. Once $v / v_{R} \geq 0.5$, a sharp transition from a single propagating MC to an ensemble of DCs (microbranches) occurs. The crack velocity therefore oscillates around 0.5 , which cannot be predicted by the classical LEFM so far [37].

In particular, we notice that the present dynamic cracking displays a so-called "directional stability". It can be seen from Fig. 2e and $f$ that the branched DC planes (marked by DC-1, DC-2, DC-3, etc.) are always parallel to the MC plane. The crack-path problem can, in principle, be treated in terms of the thermodynamic fracture criterion that proposes that the favored orientation will maximize the dynamic fracture energy [37,40]. Considering an arbitrary DC that deviates from its MC direction with a rotation $\theta$ at the branching point, then the dynamic fracture energy for this $\theta$-rotation DC is

$\Gamma(\theta)=G_{s}(\theta)\left(1-\frac{v_{b}}{c_{R}}\right)$,

where the mechanical energy release rate $G_{s}(\theta)$ is the static, time independent energy flux into the crack tip which is given by [37]

$G_{s}(\theta)=\frac{K_{\mathrm{I}}^{\prime 2}}{E /\left(1-\nu^{2}\right)}+\frac{K_{\mathrm{II}}^{\prime 2}}{E /\left(1-\nu^{2}\right)}+\frac{K_{\mathrm{III}}^{\prime 2}(1+\nu)}{E /\left(1-\nu^{2}\right)}$,

where $\nu$ is Poisson's ratio, and the transformed stress intensity factors are related to the local stress in the new crack plane, with

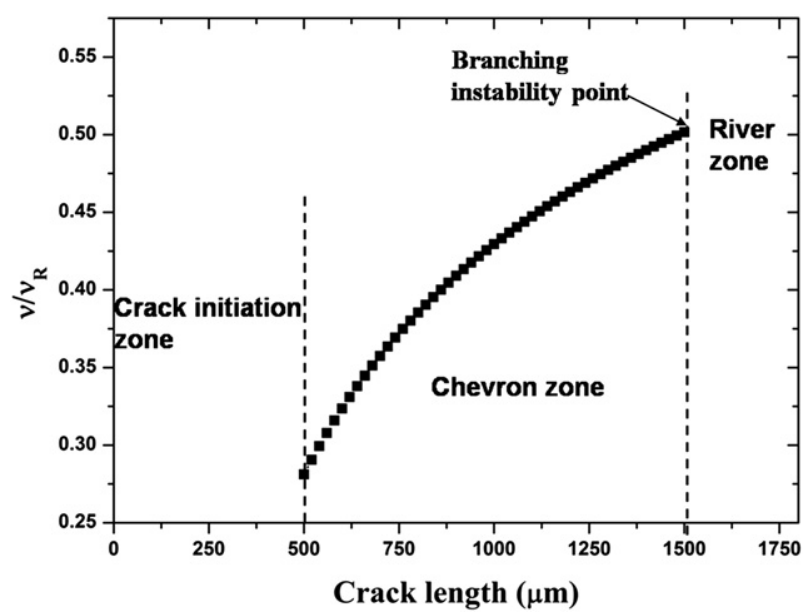

Fig. 3. Evolution of the crack velocity normalized by Rayleigh wave velocity in the chevron zone in Fig. 1d.

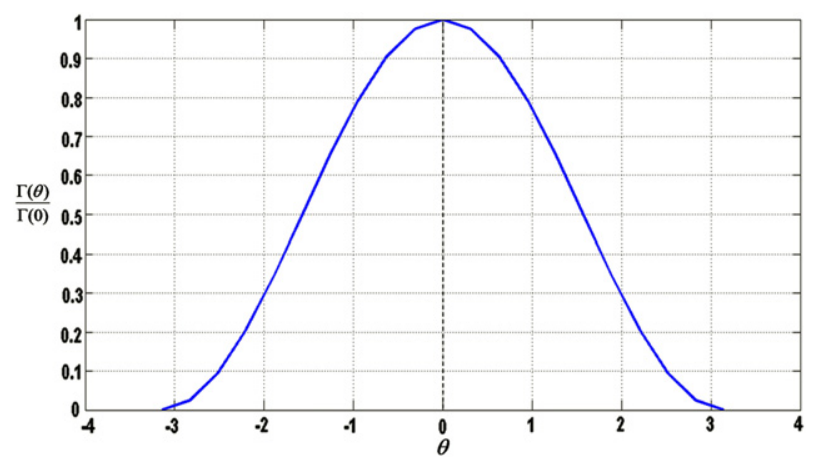

Fig. 4. Normalized dynamic fracture energy versus misorientation angle for dynamic Mode I cracking in plane stain.

$K_{\mathrm{I}}^{\prime}=\sigma_{y^{\prime} y^{\prime}} \sqrt{2 \pi r}$

$K_{\mathrm{II}}^{\prime}=\sigma_{x^{\prime} y^{\prime}} \sqrt{2 \pi r}$

$K_{\text {III }}^{\prime}=\sigma_{X^{\prime} z^{\prime}} \sqrt{2 \pi r}$.

It is noted that these stress components in Eq. (4) are just equal to those in the original polar coordinate system $(\theta, r)$ of the MC. We thus obtain the normalized dynamic fracture energy

$\frac{\Gamma(\theta)}{\Gamma(0)}=\cos ^{4}(\theta / 2)$,

where $\Gamma(0)$ is the dynamic fracture energy for original straightahead cracking. It is readily found that $\Gamma(\theta) / \Gamma(0)$ reaches the maxima at $\theta=0$, as plotted in Fig. 4. Therefore, we conclude that the dynamic branched cracks are favored to continue in their original MC plane direction (see Fig. 2e and f).

\section{Conclusions}

In summary, the fracture surface of a tough Vit 1 BMG under dynamic forced-shearing presents an interesting fractographic transition from river-like vein pattern to chevron-like zone where microscopic dimples and voids coexist, followed by crack microbranching behavior. A critical crack velocity $v / v_{R} \approx 0.5$ for the onset of the microbranching instability was determined based on our previously developed model about dynamic fracture of BMGs. In addition, it was found that the branched cracks tend to advance along the direction of their original main fracture plane.

\section{Acknowledgments}

Financial support is from the NSFC (Grants Nos. 10725211, 11002144, 11023001 and 11021262), the National Natural Science Foundation of China-NSAF. Grant No: 10976100, and the National Key Basic Research Program of China (Grant No. 2009CB724401).

\section{References}

[1] Johnson JW, Holloway DG. Philos Mag 1966;14:731.

[2] Fineberg J, Gross SP, Marder M, Swinney HL. Phys Rev Lett 1991;67:457.

[3] Ravi-Chandar K, Yang B. J Mech Phys Solids 1997;45:535.

[4] Cramer T, Wanner A, Gumbsch P. Phys Rev Lett 2000;85:788.

[5] Xi XK, Zhao DQ, Pan MX, Wang WH, Wu Y, Lewandowski JJ. Phys Rev Let 2005;94:125510.

[6] Stoica M, Eckert J, Roth S, Zhang ZF, Schultz L, Wang WH. Intermetallics 2005; 13:764.

[7] Flores KM, Dauskardt RH. Intermetallics 2004;12:1025. 
[8] Schuh CA, Hufnagel TC, Ramamurty U. Acta Mater 2007;55:4067.

[9] Dai LH, Bai YL. Int J Impact Eng 2008;35:704.

[10] Trexler MM, Thadhani NN. Prog Mater Sci 2010;55:759.

[11] Jiang F, Jiang MQ, Wang HF, Zhao YL, He L, Sun J. Acta Mater 2011;59: 2057

[12] Wu Y, Li HX, Liu ZY, Chen GL, Lu ZP. Intermetallics 2010;18:157.

[13] Schroers J, Johnson WL. Phys Rev Lett 2004;93:255506.

[14] Yavari AR, Lewandowski JJ, Eckert J. MRS Bull 2007;32:635.

[15] Yokoyama Y, Fujita K, Yavari AR, Inoue A. Phil Mag Lett 2009;89:322.

[16] Shen J, Liang WZ, Sun JF. Appl Phys Lett 2006;89:121908.

[17] Wang G, Zhao DQ, Bai HY, Pan MX, Xia AL, Han BS, et al. Phys Rev Lett 2007; 98:235501.

[18] Zhao JX, Qu RT, Wu FF, Zhang ZF, Shen BL, Stoica M, et al. J Appl Phys 2009; 105:103519.

[19] Jiang MQ Ling Z, Meng JX, Dai LH. Philos Mag 2008;88:407.

[20] Meng JX, Ling Z, Jiang MQ Zhang HS, Dai LH. Appl Phys Lett 2008;92:171909.

[21] Raghavan R, Murali P, Ramamurty U. Acta Mater 2009;57:3332.

[22] Jiang MQ, Ling Z, Meng JX, Gao JB, Dai LH. Scripta Mater 2010;62:572.

[23] Jiang MQ Meng JX, Gao JB, Wang X-L, Rouxel T, Keryvin V, et al. Intermetallics 2010;18:2468.

[24] Escobedo JP, Gupta YM. J Appl Phys 2010;107:123502.
[25] Bouchaud E, Boivin D, Pouchou J-L, Bonamy D, Poon B, Ravichandran G. Europhys Lett 2008;83:66006.

[26] Pauly S, Lee MH, Kim DH, Kim KB, Sordelet DJ, Eckert J. J Appl Phys 2009;106: 103518.

[27] Rittel D, Rosakis AJ. Eng Fract Mech 2005;72:1905.

[28] Raghavan R, Murali P, Ramamurty U. Intermetallics 2006;14:1051.

[29] Conner RD, Rosakis AJ, Johnson WL, Owen DW. Scripta Mater 1997;37:1373.

[30] Xu J, Ramamurty U, Ma E. JOM 2010;62:10.

[31] Anderson TL. Fracture mechanics: fundamentals and applications. 3rd ed. New York: CRC Press; 2005.

[32] Pampillo CA, Reimschuessel AC. J Mater Sci 1974;9:718.

[33] Keryvin V, Nadot Y, Yokoyama Y. Scripta Mater 2007;57:145.

[34] Tandaiya P, Ramamurty U, Narasimhan R. J Mech Phys Solids 2009;57:1880.

[35] Spaepen F. Acta Metall 1975;23:615.

[36] Argon AS, Salama M. Mater Sci Eng 1976;23:219.

[37] Freund LB. Dynamic fracture mechanics. Cambridge: Cambridge University Press; 1990.

[38] Wright WJ, Hufnagel TC, Nix WD. J Appl Phys 2003;93:1432.

[39] Flores KM, Dauskardt RH. Acta Mater 2001;49:2527.

[40] Lawn BR, Wilshaw TR. Fracture of brittle solids. Cambridge: Cambridge University Press; 1975. 